\title{
Histone Deacetylase Inhibitor Vorinostat (SAHA) Suppresses IL-1 $\beta$-Induced Matrix Metallopeptidase-13 Expression by Inhibiting IL-6 in Osteoarthritis Chondrocyte
}

\author{
Mohammad Shahidul Makki and Tariq M. Haqqi
}

From the Department of Anatomy and Neurobiology, Northeast Ohio Medical University, Rootstown, Ohio

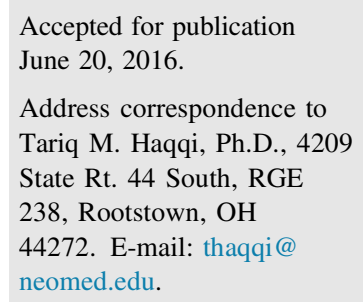

\begin{abstract}
Osteoarthritis $(0 \mathrm{~A})$ is the most common whole-joint disease and is characterized by progressive loss of the cartilage matrix. Matrix metallopeptidase-13 (MMP-13) is a highly active and an abundantly expressed protease in $\mathrm{OA}$ cartilage and chondrocytes and degrades type II collagen and proteoglycans. We investigated the mechanism of MMP-13 suppression by histone deacetylase inhibitor vorinostat (SAHA). OA chondrocytes were obtained from knee cartilage after enzymatic digestion and treated with IL-1 $\beta$ in the absence or presence of various histone deacetylase inhibitors. Gene expression was quantified using quantitative RT-PCR. Protein expression and chromatin modifications were determined by Western immunoblotting using specific antibodies. The effect of IL- 6 on the expression of MMP-13 was determined by treating chondrocytes with recombinant IL- 6 or by IL6 knockdown using IL6-specific siRNA. We found that SAHA is a potent suppressor of IL-1 $\beta$-induced MMP-13, tumor necrosis factor- $\alpha$, and other catabolic marker expression in $0 A$ chondrocytes. Interestingly, SAHA rescued the COL2A1 and ACAN expression in $O A$ chondrocytes that was down-regulated by IL-1 $\beta$. Of importance is our finding that IL-6-stimulated MMP-13 expression was independent of IL-1 $\beta$ stimulation and was blocked by SAHA, suggesting that SAHA inhibits IL- 6 signaling in OA chondrocytes. Taken together, our results suggest that SAHA could be used as a therapeutic agent for the management of OA. (Am J Pathol 2016, 186: 2701-2708; http:// dx.doi.org/10.1016/j.ajpath.2016.06.010)
\end{abstract}

Osteoarthritis $(\mathrm{OA})$ is a degenerative disease of the whole joint and is characterized by progressive loss of the cartilage matrix. Other characteristics include osteophyte formation, persistent loss of tissue cellularity, changes in periarticular cartilage, and remodeling of the subchondral bone. Joint discomfort compromises quality of life and leads to lost productivity, especially in the elderly population. Particular attention is dedicated to the cartilage in OA because of the observed degenerative loss of cartilage integrity that is central to the pathogenesis of the disease. ${ }^{1-3}$ The chondrocyte is the only cell type present in the cartilage and is critical for tissue homeostasis through measured production of anabolic and catabolic factors. It is now agreed that a critical factor in OA pathogenesis is the excessive production of proinflammatory cytokines including IL-1 $\beta$, a key player in the disease pathogenesis. ${ }^{4,5}$ Noticeably, IL-1 $\beta$ is an inducer of expression of matrix metallopeptidases (MMPs) and members of the ADAMTs family of proteases in chondrocytes and a suppressor of cartilage matrix formation. ${ }^{6}$ Among the MMPs, MMP-13 is highly active and an abundantly expressed protease in late-stage OA chondrocytes compared with early stage OA or normal healthy chondrocytes. ${ }^{7}$ MMP-13 is active against type II, IV, and IX

Supported in part by United States Public Health Service/NIH grants (RO1 AT007373, RO1 AT005520, RO1 AR067056, and R21 AR064890) and funds from North East Ohio Medical University (T.M.H.).

Disclosures: None declared. 
collagen, and also degrades proteoglycans, osteonectin, and perlecan in cartilage and thus serves as a key protease involved in cartilage degradation. ${ }^{7}$ Transgenic mice with targeted overexpression of Mmp-13 in articular cartilage develop a spontaneous OA-like phenotype, ${ }^{8}$ whereas $M M P$ 13 knock-out mice are resistant to osteoarthritic cartilage damage, suggesting that MMP-13 activity significantly contributes to cartilage erosion in OA. ${ }^{9}$

For efficient gene regulation, nucleosomal histone proteins undergo post-translational modifications. ${ }^{10}$ One of the most-studied modifications that affects the gene regulatory process enormously is acetylation and deacetylation of core histone proteins. This is accomplished by two different groups of enzymes: namely, histone acetyltransferases and histone deacetylases (HDACs). HDACs catalyze the removal of the acetyl group from the histone protein and repress gene activation. ${ }^{11,12}$ The HDAC family has been grouped into three classes: class I HDACs include HDAC-1, $-2,-3$, and -8 and are related to yeast RPD3; class II HDACs include HDAC-4, -5, -6, -7, -9, and -10 and are closely related to yeast HDA1; and class III HDACs are dependent on the oxidized form of nicotinamide-adenine dinucleotide and are homologs of yeast Sir2 protein. HDAC inhibitors (HDACi) block the activity of HDAC enzymes and reverse the deacetylation process. ${ }^{13}$ HDACi have been reported to modulate the expression of proinflammatory cytokines and catabolic proteases and have been used in an experimental model of arthritis with positive outcomes. ${ }^{14-17}$ In this study we found that vorinostat (SAHA, a class I and II HDAC inhibitor) blocks the IL-1 $\beta$-induced expression of MMP-13 in human OA chondrocytes. Furthermore, we investigated the mechanism of SAHA-mediated inhibition of MMP-13 expression in human OA chondrocytes and discovered that it is mediated, at least in part, through the suppression of IL-6 expression.

\section{Materials and Methods}

\section{Reagents}

CellGro ACTive media was procured from CellGenix (cat. 24804-0500; Frieburg, Germany). Dulbecco's modified Eagle medium (DMEM), fetal bovine serum (cat: SH30243FS), High-Capacity cDNA Reverse Transcription Kit (cat: 4368814), and TaqMan Gene Expression Assays were purchased from Thermo Fisher/Life Technologies (Carlsbad, CA). For enzymatic digestion of cartilage, pronase (cat: 11459643001) and collagenase (cat: 11088815001) were obtained from Roche Diagnostics (Indianapolis, IN). RNA isolation was performed using Qiazol and the miRNeasy kit procured from Qiagen (cat: 217004; Valencia, CA). Recombinant human IL-1 $\beta$ (cat: 201-LB-025), soluble IL-6 receptor (sIL-6R; cat: cyt-286-b), and IL-6 (cat: 206-IL/CF) were obtained from Biotechne/R\&D Systems (Minneapolis, MN). Antibodies against $\beta$-actin (cat: sc-47778) and MMP-13 (cat: sc-30073) were purchased from Santa Cruz Biotechnology
(Santa Cruz, CA). Anti-IL-6 (cat: 12153), anti-Ac-H4 (cat: 9672), and H4 (cat: 2592) antibodies were obtained from Cell Signaling Technology (Danvers, MA). Horseradish-peroxidase-conjugated anti-mouse (cat: 1858413) and anti-rabbit (cat: 32460) secondary antibodies were obtained from Pierce Biotechnology (Rockford, IL). HDAC inhibitors SAHA (cat: s1047), Trichostatin A (TSA; cat: s1045), Val Proic Acid (VPA; cat: 1168), and MS-275 (cat: s1053) were purchased from Selleckchem (Houston, TX).

\section{Cartilage and Chondrocyte Preparation}

The Institutional Review Board of North East Ohio Medical University (Rootstown, $\mathrm{OH}$ ) and SUMMA Health Systems (Akron, $\mathrm{OH}$ ) approved the study protocol as "not a human subject study under 45 CFR [The Code of Federal Regulations]." Discarded and de-identified cartilage samples were obtained from donors who underwent total knee replacement surgery because of degenerative joint disease and were between 48 and 71 years of age $(n=12$; mean age, $55 \pm 12.4$ years; 5 men and 12 women). Cartilage was carefullyexcised aseptically using a sterile scalpel blade from the smooth areas (no staining with India ink; Mankin score, 1) and half of the cartilage was stored immediately in liquid nitrogen for later use. The remaining cartilage was used for chondrocyte preparation using enzymatic digestion as previously described. ${ }^{18,19}$

\section{Chondrocyte Treatment}

For each treatment, $1.2 \times 10^{6}$ primary OA chondrocytes were seeded into $3.5-\mathrm{cm}$ dishes in complete DMEM. When $80 \%$ confluent, the medium was changed and the chondrocytes were incubated in CellGro ACTive medium without serum for 24 hours. For HDAC inhibitor experiments, chondrocytes first were induced with $2 \mathrm{ng} / \mathrm{mL} \mathrm{IL}-1 \beta$ for 2 hours, and then treated with HDAC inhibitors $(500$ $\mathrm{nmol} / \mathrm{L}$ TSA, $1 \mu \mathrm{mol} / \mathrm{L} \mathrm{SAHA}, 1 \mathrm{ng} / \mathrm{mL}$ VPA, and $1 \mu \mathrm{mol} /$ L MS-275) for an additional 14 hours. After treatment, chondrocytes were washed with phosphate-buffered saline and RNA, protein was prepared immediately, or cells were stored at $-80^{\circ} \mathrm{C}$ for later use. For IL-6 treatment, chondrocytes were incubated in CellGro ACTive medium as described earlier and treated with different concentrations of IL-6 for 24 hours. In the experiments in which OA chondrocytes were treated sequentially with IL-6 and SAHA, chondrocytes first were incubated with IL- 6 for 12 hours, then the medium was supplemented with SAHA and the incubation continued for an additional 12 hours.

\section{Treatment of Cartilage Explants}

Cartilage explants were prepared from the OA cartilage samples $(n=5 ; 22.5 \pm 4.2 \mathrm{mg})$ using a sterile number 10 Feather scalpel blade (Feather Safety Razor Co., Osaka, Japan) and were incubated in a 24-well plate using DMEM 
supplemented with $10 \%$ fetal bovine serum. After 24 hours the medium was changed to serum-free CellGro ACTive medium supplemented with $2.0 \mathrm{ng} / \mathrm{mL}$ IL- $1 \beta$ alone. After 2 hours of treatment with IL-1 $\beta$ the explants were left untreated or the medium was supplemented with $1.0 \mu \mathrm{mol} / \mathrm{L}$ SAHA and the incubation was continued for an additional 24 hours. At the end of the experiment RNA was prepared from cartilage explants as described later.

\section{Transfection of OA Chondrocytes with siRNAs}

For knock-down of target mRNA, $5 \times 10^{6}$ OA chondrocytes were seeded in a $10-\mathrm{cm}$ petri dish in complete DMEM and when $80 \%$ confluent were transfected with siRNA targeted against IL-6 or control nontargeted siRNA at a final concentration of $100 \mathrm{nmol} / \mathrm{L}$ using the Amaxa Nucleofactor kit (Amaxa, Gaithersburg, MD) according to the manufacturer's recommendations. Briefly, adherent chondrocytes were digested with $1 \mathrm{mg} / \mathrm{mL}$ pronase and collagenase for 3 hours. IL6 siRNA or nontargeted siRNA was diluted to $100 \mu \mathrm{L}$ in nucleofactor solution and chondrocytes were transfected using electroprogramme P01. Chondrocytes then were seeded in DMEM supplemented with $10 \%$ fetal bovine serum and 24 hours later the culture medium was changed to serum-free CellGro ACTive medium, and after 12 hours the chondrocytes were treated with $2 \mathrm{ng} / \mathrm{mL} \mathrm{IL-1 \beta}$ for 24 hours in the same medium.

\section{Preparation of Total RNA and Gene Expression Analysis}

Total RNA from cultured chondrocytes was prepared by lyzing the cells directly in the lysis buffer (RNeasy Plus mini kit) and RNA was prepared essentially as described in the protocol provided with the kit. For preparing the total RNA from the explants, control and treated cartilage explants were ground to a fine powder with a steel mortar and pestle in liquid nitrogen to prevent RNA degradation. Powdered cartilage was transferred into $6 \mathrm{~mL}$ Qiazol solution, the solution was vortexed, and then was divided into three 2-mL Eppendorf tubes. After the addition of $200 \mu \mathrm{L}$ of chloroform, the aqueous phase from each tube was pooled (approximately $4 \mathrm{~mL}$ ) and divided into two tubes $(2 \mathrm{~mL} /$ tube), and subsequently transferred onto a RNeasy Mini Spin column (Qiagen). DNA was digested on the column and the DNA-free RNA was eluted in RNAsefree water as per the instructions provided with the kit. RNA quality and quantity was determined by the NanoDrop 2000c (Thermo Fisher Scientific, Waltham, MA).

Single-stranded cDNA was synthesized using the genomic DNA-free total RNA prepared as previously described using $1.0 \mu \mathrm{g}$ total RNA and the High-Capacity cDNA Reverse Transcription Kit. ${ }^{20}$ Gene expression levels of COL2A1 (NM_001844), Aggrecan (NM_013227), COL10A1 (NM_ 008032), COL1A1 (NM_000088), MMP-2 (NM_001302510), MMP-3 (NM_002422), MMP-9 (NM_004994), MMP-13 (NM_002427), ADAM17 (NM_00318), ADAMTS-5
(NM_007038), tumor necrosis factor- $\alpha$ (X02910), and IL-6 (NM_000600) was quantified using TaqMan assays (Life Technologies). Expression levels were determined in one plate for each set of experiments. Values were normalized to the corresponding $\beta$-actin expression level measured within the same plate. Relative expression levels were calculated using the $2^{-\Delta \Delta \mathrm{CT}}$ method. $^{21}$

\section{Immunoblot Analysis}

After the desired treatment, OA chondrocytes were harvested, washed with phosphate-buffered saline, and lyzed in ice-cold protein lysis buffer $(50 \mathrm{ng} / \mathrm{mL}$ Tris $\mathrm{pH} \mathrm{7.6,} 400$ $\mathrm{ng} / \mathrm{mL} \mathrm{NaCl}, 0.5 \% \mathrm{NP}-40,1 \mathrm{ng} / \mathrm{mL}$ phenylmethylsulfonyl fluoride, and $1 \times$ protease inhibitor cocktail; Roche, Mannheim, Germany). ${ }^{18,22}$ Lysates were cleared by centrifugation $\left(15,000 \times g\right.$ for 10 minutes at $\left.4^{\circ} \mathrm{C}\right)$ and analyzed immediately or stored at $-80^{\circ} \mathrm{C}$. Equivalent amounts of proteins were resolved by $10 \%$ SDS-PAGE and transferred onto a polyvinylidene difluoride membrane (Immobilon-P Membrane, cat: IPVH00010; EMD Millipore, Billerica, MA). After blocking in 5\% fat-free milk, membranes were incubated with anti-IL-6 (1:1000), anti-MMP-13, or anti- $\beta$ actin (1:5000) primary antibodies overnight at $4^{\circ} \mathrm{C}$. To detect acetylated histone, cells were lyzed in protein loading buffer ( $0.313 \mathrm{~mol} / \mathrm{L}$ Tris $\mathrm{pH} 6.8,10 \% \mathrm{SDS}, 40 \%$ glycerol, $0.05 \%$ bromophenol blue, and $2 \% \beta$-mercaptoethanol). Cells were sonicated at $50 \%$ intensity three times for 10 seconds each using a probe-based sonicator (model FB705; Fisher Scientific, Pittsburgh, PA), centrifuged, and the cleared lysate was separated on $12 \%$ SDS-PAGE. Afterward, blocking membranes were incubated with anti-Ac-H4 (1:1000) or anti-H4 (1:1000) antibodies overnight at $4^{\circ} \mathrm{C}$. For detecting the immune-reactive proteins, membranes were probed using appropriate horseradish-peroxidase-conjugated secondary antibody (1:2000), and the protein bands were visualized using Syngene PXi 4 imager (Syngene, Frederick, MD). Quantification of immunoreactive protein bands was performed using Gene Tools Software (Syngene).

\section{Statistical Analysis}

The results are presented as the means $\pm \mathrm{SD}$. The significant difference between the experimental groups and controls was assessed by $t$-test. Each experiment was repeated three times using three independent patient samples. The difference was considered significant if the $P<0.05$.

\section{Results}

MMP-13 Expression in OA Chondrocytes Treated with HDAC Inhibitor

To assess the effect of HDAC inhibitors on the expression of MMP-13 in OA chondrocytes, we used HDACi specific for either class I or both classes I and II. TSA and SAHA could 
A

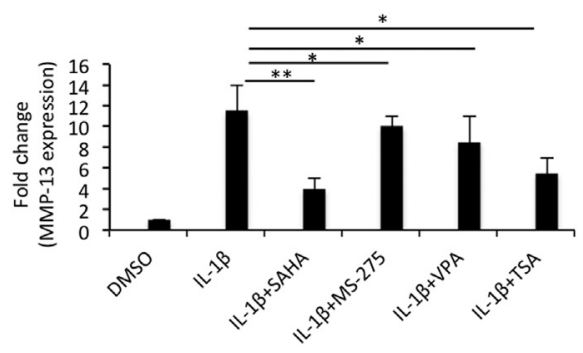

C

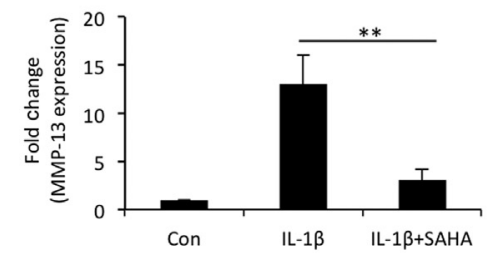

B

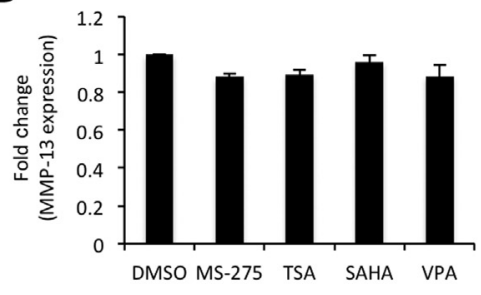

D

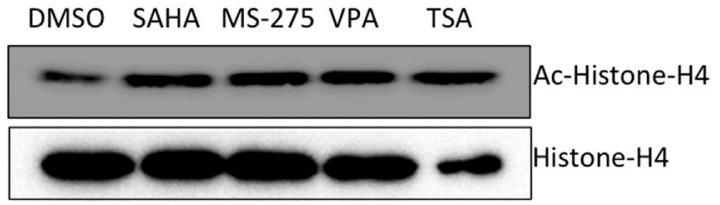

Figure 1 SAHA is a potent suppressor of matrix metallopeptidase (MMP)-13 expression in IL-1 $\beta$-stimulated osteoarthritis (0A) chondrocytes. A: 0A chondrocytes were induced with $1 \mathrm{ng} / \mathrm{mL} \mathrm{IL-} 1 \beta$ for 2 hours and then treated with different histone deacetylase (HDAC) inhibitors ( $1 \mu \mathrm{mol} / \mathrm{L} \mathrm{SAHA}, 1 \mu \mathrm{mol} / \mathrm{L} \mathrm{MS}$ $275,1 \mathrm{ng} / \mathrm{mL} \mathrm{VPA}$, and $500 \mathrm{nmol} / \mathrm{L} \mathrm{TSA}$ ) for an additional 14 hours in serum-free CellGro Active Chondrocyte Culture Medium (CellGenix). Chondrocytes were harvested, total RNA was prepared, and MMP-13 expression was quantified by TaqMan assay (Thermo Fisher/Life Technologies). B: Chondrocytes were cultured in medium containing serum and treated with different HDAC inhibitors. Sixteen hours later the chondrocytes were harvested and basal expression of MMP-13 mRNAs was detected by TaqMan assay. C: Control or treated cartilage explant samples were used to isolate total RNA and the TaqMan assay was used to quantify the expression of MMP-13 mRNA. D: Hyperacetylation of histone 4 was detected by anti-acetyl-H4 antibody. Data are presented as means \pm SD of independent experiments using five patients' cartilage samples. ${ }^{*} P<0.05,{ }^{* *} P<0.005$. Con, control; DMSO, dimethyl sulfoxide.

inhibit the class I as well as class II, but VPA and MS-275 blocked the activity of class I HDACs only. All four HDAC inhibitors down-regulated the IL-1 $\beta$-induced expression of MMP-13, but the effect was more robust with SAHA (Figure 1A). Therefore, we used SAHA in all of our subsequent experiments. Basal expression of MMP-13 in OA chondrocytes remained unchanged upon HDAC inhibitor treatment, suggesting that these inhibitors suppressed the IL$\beta$-induced MMP-13 expression in chondrocytes, possibly through interference with cytokine-stimulated chromatin remodeling (Figure 1B). Similar suppression of MMP-13 expression was observed when cartilage explants were treated with SAHA and stimulated with IL-1 $\beta$ (Figure 1C). These data suggest that SAHA effectively reduced the expression of MMP-13 in IL-1 $\beta$-treated OA chondrocytes and the OA cartilage explants in vitro. In a control experiment, inhibition of HDAC was shown by hyperacetylation of histone H4 (Figure 1D).
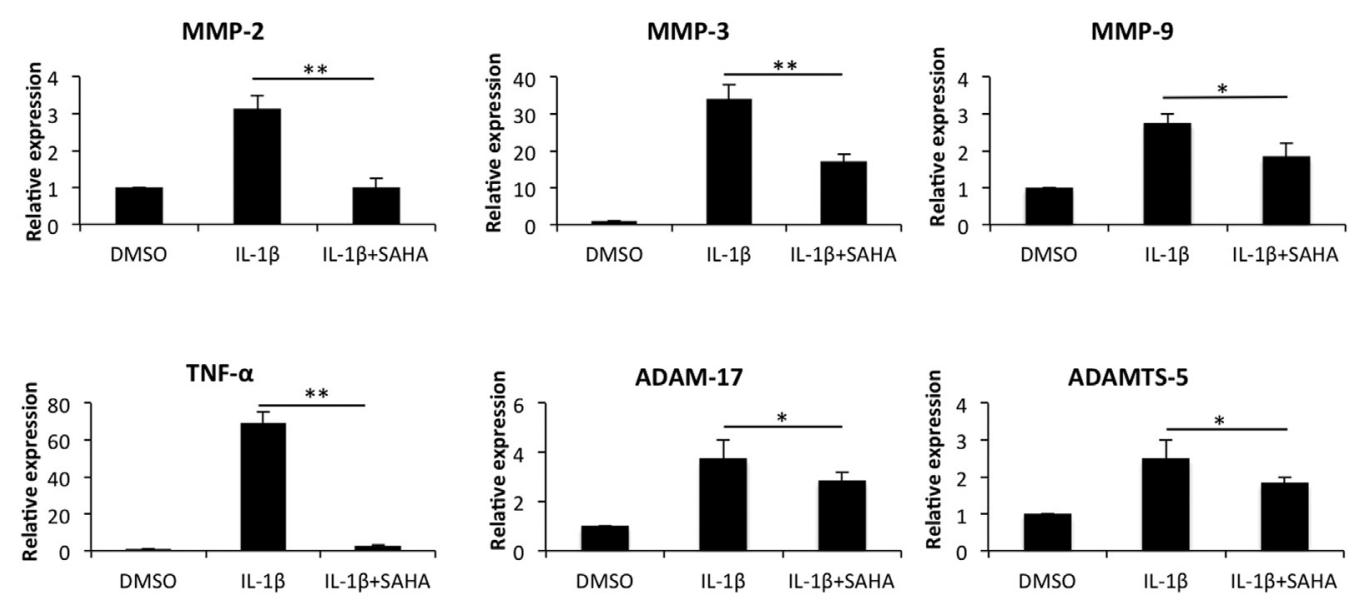

Figure 2 SAHA abolishes the expression of tumor necrosis factor (TNF)- $\alpha$ and inhibits the expression of selected catabolic markers in IL-1 $\beta$-treated osteoarthritis (OA) chondrocytes. OA chondrocytes were induced with $2 \mathrm{ng} / \mathrm{mL} \mathrm{IL-1 \beta}$ for 2 hours and then treated with $1 \mu \mathrm{mol} / \mathrm{L}$ SAHA for an additional 14 hours in serum-free CellGro Active chondrocyte culture medium (CellGenix). Chondrocytes were harvested and total RNA was prepared. Expression of matrix metallopeptidase (MMP)-2, MMP-3, MMP-9, tumor necrosis factor (TNF)- $\alpha$, ADAM-17, and ADAMTS- 5 was quantified by TaqMan assays (Thermo Fisher/Life Technologies). ${ }^{*} P<0.05,{ }^{*} P<0.005$. DMSO, dimethyl sulfoxide. 

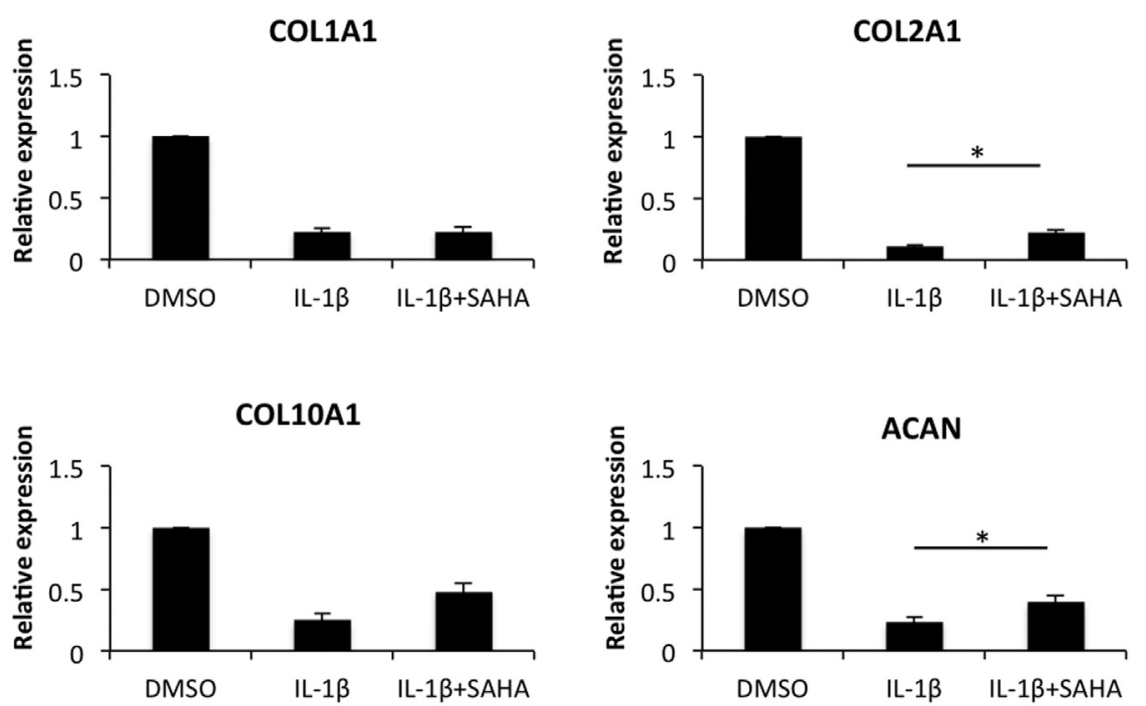

Figure 3 Treatment with SAHA reduces the expression of anabolic genes in IL-1 $\beta$-induced osteoarthritis $(0 \mathrm{~A})$ chondrocytes. OA chondrocytes were induced with $2 \mathrm{ng} / \mathrm{mL}$ IL-1 $\beta$ for 2 hours and then treated with $1 \mu \mathrm{mol} / \mathrm{L}$ SAHA for an additional 14 hours in serum-free CellGro Active chondrocyte culture medium. RNA was prepared from treated chondrocytes and single-stranded cDNA was synthesized and was used to quantify the expression of COL1A1, COL2A1, COL10A1, and aggrecan (ACAN) by TaqMan assay (Thermo Fisher/Life Technologies). ${ }^{*} P<0.05$. DMSO, dimethyl sulfoxide.
Treatment with SAHA Suppresses the Expression of Catabolic Markers but Enhances the Expression of Anabolic Markers

We first estimated the effect of SAHA on the levels of MMP2 , MMP-3, MMP-9, tumor necrosis factor- $\alpha$, ADAM-17, and ADAMTS-5 in IL-1 $\beta$-stimulated OA chondrocytes. When OA chondrocytes were treated with IL- $\beta$ alone, significant induction of all the catabolic genes, albeit with varying degrees, was observed (Figure 2). This enhanced expression of catabolic genes by IL-1 $\beta$ was suppressed significantly by SAHA (Figure 2). The most dramatic reduction was observed in case of IL-1 $\beta$-induced expression of tumor necrosis factor- $\alpha$, which was reduced from a 70 -fold increase to approximately a fivefold increase compared with the chondrocytes treated with IL-1 $\beta$ alone (Figure 2). All other genes also showed a significant reduction, ranging from an approximately $30 \%$ to $70 \%$ reduction compared with the levels detected in chondrocytes treated with IL-1 $\beta$ alone (Figure 2). We also were interested in determining the impact of treatment with SAHA on the expression of anabolic genes in OA chondrocytes. As reported previously in several publications, IL-1 $\beta$ treatment down-regulated the expression of COL1A1, COL2A1, COL10A1, and aggrecan (ACAN) in OA chondrocytes. ${ }^{6,23}$ Interestingly, we found that treatment with SAHA expression of COL2A1 and ACAN was increased significantly $(P<0.05)$ compared with chondrocytes treated with IL-1 $\beta$ alone (Figure 3 ).

\section{Effect of IL-6 on MMP-13 Expression}

As reported previously, treatment with IL-1 $\beta$ induced the high level expression of IL-6 and MMP-13 in human OA chondrocytes. ${ }^{24}$ We previously showed that the expression of IL-6 precedes the expression of MMP-13 in IL$1 \beta$-stimulated human OA chondrocytes, ${ }^{24}$ although the relationship was not clear. Therefore, we investigated whether induction of MMP-13 is mediated by IL-6 in human OA chondrocytes. We treated the cultured OA chondrocytes with different concentrations of recombinant human IL-6 plus $50 \mathrm{ng} / \mathrm{mL}$ sIL-6R. Expression of MMP-13 was increased threefold at a $10 \mathrm{ng} / \mathrm{mL}$ concentration of IL-6 and showed no further increase with the increasing concentrations of IL-6 (Figure 4), suggesting that higher concentrations of IL-6 were not effective in further augmenting the expression of MMP-13 in OA chondrocytes.

\section{siRNA-Mediated Knockdown of IL-6 Reduces the Expression of MMP-13}

To establish the connection between IL-6 and MMP-13 we performed siRNA-mediated knockdown of IL-6 expression in human OA chondrocytes. IL-1 $\beta$-induced IL-6 mRNA expression was $90 \%$ reduced in OA chondrocytes transfected with a IL-6-specific siRNA compared with the levels detected in nontargeted siRNA-transfected OA chondrocytes (Figure 5A). Knockdown of IL-6 expression correlated with

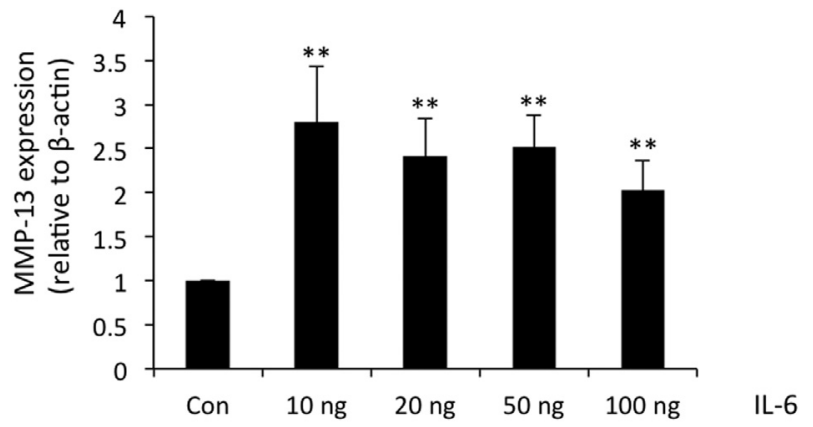

Figure 4 Expression of matrix metallopeptidase (MMP)-13 upon IL-6 and sIL-6R treatment in osteoarthritis $(0 \mathrm{~A})$ chondrocytes. OA chondrocytes were treated with different concentrations of IL- 6 in the presence of fixed concentration of sIL-6R $(50 \mathrm{ng} / \mathrm{mL})$ for 24 hours. Total RNA and CDNA was prepared from control and IL-6-treated chondrocytes as described earlier. Expression of MMP-13 was measured by TaqMan assay (Thermo Fisher/Life Technologies). ${ }^{*} P<0.005$. Con, control; sIL-6R, soluble IL-6 receptor. 
A

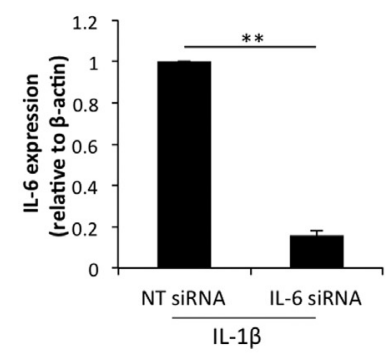

B

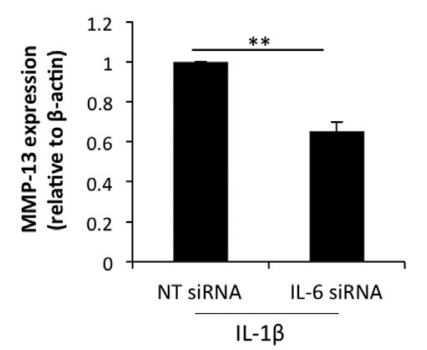

C NT SIRNA IL-6 siRNA
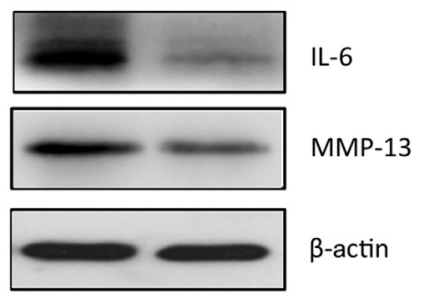

Figure 5 siRNA-mediated depletion of interleukin (IL)- 6 inhibits the expression of matrix metallopeptidase (MMP)-13 in IL-1 $\beta$-stimulated osteoarthritis $(0 A)$ chondrocytes. A and B: OA chondrocytes were transfected with IL-6-specific siRNA for 24 hours and induced with IL- $\beta$ for an additional 24 hours. Expression of IL-6 (A) and MMP-13 (B) mRNA was measured by TaqMan assay. C: IL-6 and MMP-13 protein levels in IL-6 knockdown chondrocytes was measured by Western blot analysis using anti-IL- 6 and anti-MMP-13 antibodies. $\beta$-Actin was used as a loading control. ${ }^{* * P}<0.005$. NT, non-targeted.

the reduced expression of MMP-13 mRNA (Figure 5B) in IL-1 $\beta$-stimulated OA chondrocytes. We performed Western blot analysis to determine the effect of IL- 6 mRNA knockdown on IL-6 and MMP-13 protein expression in OA chondrocytes. Protein levels of IL-6 as well as of MMP-13 were reduced significantly in OA chondrocytes with siRNAmediated depletion of IL-6 expression (Figure 5C). This indicated that in IL- $\beta$-induced expression of MMP-13, expression of IL-6 is critical for the enhanced expression of MMP-13 in OA chondrocytes.

\section{SAHA Down-Regulates IL-6-Induced MMP-13 Expression}

To determine whether SAHA inhibits IL-6-induced expression of MMP-13, we treated OA chondrocytes with IL-6 + sIL-6R or in combination with SAHA. IL6-induced expression of MMP-13 was reduced approximately threefold upon SAHA treatment (Figure 6). These data suggest that SAHA interferes with IL-6-mediated signaling events required for MMP-13 induction and expression in $\mathrm{OA}$ chondrocytes.

\section{MMP-13}

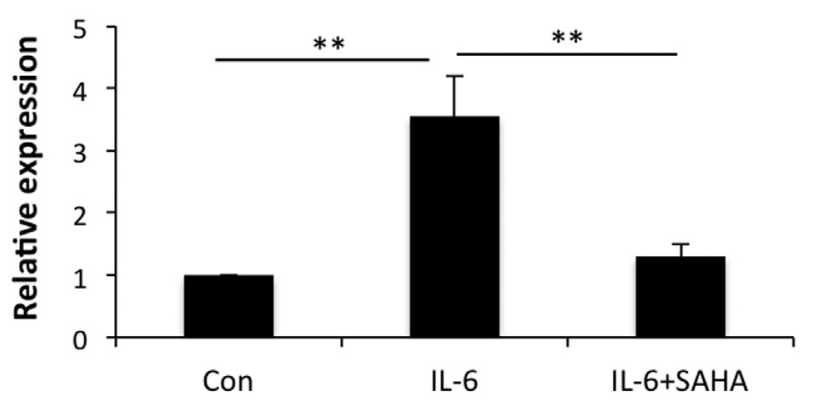

Figure 6 IL-6 induces matrix metallopeptidase (MMP)-13 expression upon SAHA treatment. Cultured chondrocytes were treated with $10 \mathrm{ng} / \mathrm{mL}$ $\mathrm{IL}-6+50 \mathrm{ng} / \mathrm{mL}$ sIL-6R for 12 hours and then supplemented with $1 \mu \mathrm{mol} / \mathrm{L}$ SAHA for an additional 12 hours. Chondrocytes were harvested and subsequently RNA and CDNA were prepared. MMP-13 expression was measured using TaqMan (Thermo Fisher/Life Technologies) real-time PCR assay. ${ }^{*} P<0.005$. Con, control; sIL-6R, soluble IL-6 receptor.

\section{Discussion}

Here, we explored the effect of HDACi SAHA on IL-1 $1 / \mathrm{IL}-$ 6-induced expression of anabolic and catabolic genes, particularly of MMP-13 in OA chondrocytes. We showed here that class I as well as classes I and II HDACi significantly reduced the IL-1 $\beta$-induced expression of MMP-13 in OA chondrocytes. Among the HDACi tested, treatment with SAHA was found to be more pronounced in suppressing the cytokine-induced expression of MMP-13 in OA cartilage and chondrocytes. All the analyzed catabolic genes induced by IL- $1 \beta$ were inhibited significantly upon SAHA treatment, whereas expression of COL2A1 and ACAN was up-regulated significantly. Importantly, our data also showed that in IL-1 $\beta$-induced expression of MMP-13, expression of IL-6 is a critical factor based on the data that depletion of IL-6 expression significantly affects the expression of MMP-13 in IL-1 $\beta$-stimulated OA chondrocytes. Although previous studies reported an antiinflammatory effect of $\mathrm{HDACi},{ }^{15,17,25}$ this is the first report to also show that IL-6-induced MMP-13 expression was suppressed by SAHA in OA chondrocytes, suggesting that SAHA may be a suppressor of signaling events activated by IL- 6 in OA chondrocytes.

The destructive character of $\mathrm{OA}$ prevails owing to persistent expression of proinflammatory cytokines by activated synovium and hypertrophic chondrocytes. The irreversible nature of the cartilage damage synergizes with the presence of high levels of metalloproteinases and ADAMTs family of proteins in the OA joint. Because current treatments are mostly symptomatic and rely on nonsteroidal anti-inflammatory drugs to alleviate the discomfort, potential cure or significant inhibition of OA progression still is not feasible as a result of a lack of approved treatment and delivery regimens that can modify the disease progression in OA. Histone deacetylases posttranslationally modify histone and nonhistone proteins and determine gene expression outcome. ${ }^{26}$ Global HDAC activities are increased in rheumatoid arthritis patients when compared with healthy individuals. ${ }^{25}$ Expression of HDACs 1 and 2 was found to be increased in the osteoarthritic 
condition, suggestive of a potential role in disease pathogenesis. ${ }^{27}$ The expression of HDAC4 was increased in OA chondrocytes, whereas knockdown of HDAC4 attenuated the induction of catabolic gene expression in chondrocytes. ${ }^{28}$ In another study, direct correlation was observed in the enhanced expression of MMP-13 and increased HDAC7 levels in OA cartilage. ${ }^{29}$ Treatment with TSA, valproic acid, or MS-275 blocks the cytokine-induced expression of MMP-1 and -13 in human articular chondrocytes. ${ }^{30}$ Another HDACi, ITF2357, which inhibits class I and II HADCs, reduced the expression of proinflammatory cytokines in synovial tissues, but the mechanisms are not fully clear. ${ }^{31,32}$ Therefore, it is plausible that the inhibition of HDACs may have a beneficial effect on the management of OA. Indeed, various studies have shown the protective effect of HDACi on cartilage degeneration using a collagen antibody-induced arthritis or destabilization of the medial meniscus mouse model of OA. ${ }^{30,31}$

To our knowledge, all of the reported HDACi have been shown to down-regulate catabolic gene expression in chondrocytes but are not able to alter anabolic gene expression, especially the expression of COL2A1 and ACAN. In this study, we found that SAHA could potentially restore the chondrocyte anabolic gene expression by downregulating the inflammatory cytokine and MMP expression and up-regulating the expression of anabolic markers COL2A1 and ACAN under pathologic conditions. However, it also was very intriguing that all the tested HDACi including SAHA did not alter the basal expression of MMP13 in chondrocytes, suggesting that SAHA treatment may not affect the physiological levels of MMP-13 in normal cells. However, this needs to be investigated further.

IL-1 $\beta$-induced MMP-13 expression is regulated by p38 mitogen-activated protein kinase and the Janus kinase/ STAT pathways. ${ }^{33}$ MMP-13 expression in chondrocytes also is modulated by the c-Jun N-terminal kinase and NF$\kappa \mathrm{B}$ signaling pathways. ${ }^{34}$ Previous studies have shown that SAHA selectively attenuates mitogen-activated protein kinase and NF- $\kappa \mathrm{B}$ signaling activity in IL-1 $\beta$-induced human OA chondrocytes. ${ }^{35}$ We did not study the signaling events in the present study but it is known that IL-6 signals through activation of both STAT and mitogen-activated protein kinase pathways. ${ }^{36,20}$ Specific inhibitors of the STAT pathway block MMP production in IL-6-stimulated chondrocytes. ${ }^{23}$ Taken together, our data showing that SAHA blocks IL6-induced MMP-13 expression suggest it also could attenuate the JAK/STAT pathway in human OA chondrocytes.

In conclusion, we show for the first time that IL$1 \beta$-induced expression of IL- 6 is a critical factor in the expression of MMP-13 in OA chondrocytes. Our data also suggest that SAHA-mediated suppression of MMP-13 expression also may be through the suppression of IL-6 expression in OA under pathologic conditions. This suggests that SAHA could be developed as a therapeutic agent for the management of OA.

\section{References}

1. Goldring MB, Goldring SR: Osteoarthritis. J Cell Physiol 2007, 213 : 626-634

2. Wang M, Shen J, Jin H, Im HJ, Sandy J, Chen D: Recent progress in understanding molecular mechanisms of cartilage degeneration during osteoarthritis. Ann N Y Acad Sci 2011, 1240:61-69

3. Loeser RF, Goldring SR, Scanzello CR, Goldring MB: Osteoarthritis: a disease of the joint as an organ. Arthritis Rheum 2012, 64: 1697-1707

4. Loeser RF: Molecular mechanisms of cartilage destruction: mechanics, inflammatory mediators, and aging collide. Arthritis Rheum 2006, 54: $1357-1360$

5. Goldring SR, Goldring MB: The role of cytokines in cartilage matrix degeneration in osteoarthritis. Clin Orthop Relat Res 2004, 427 Suppl: S27-S36

6. Sandell LJ, Xing X, Franz C, Davies S, Chang LW, Patra D: Exuberant expression of chemokine genes by adult human articular chondrocytes in response to IL-1beta. Osteoarthritis Cartilage 2008, $16: 1560-1571$

7. Bau B, Gebhard PM, Haag J, Knorr T, Bartnik E, Aigner T: Relative messenger RNA expression profiling of collagenases and aggrecanases in human articular chondrocytes in vivo and in vitro. Arthritis Rheum 2002, 46:2648-2657

8. Neuhold LA, Killar L, Zhao W, Sung ML, Warner L, Kulik J, Turner J, Wu W, Billinghurst C, Meijers T, Poole AR, Babij P, DeGennaro LJ: Postnatal expression in hyaline cartilage of constitutively active human collagenase-3 (MMP-13) induces osteoarthritis in mice. J Clin Invest 2001, 107:35-44

9. Little CB, Barai A, Burkhardt D, Smith SM, Fosang AJ, Werb Z, Shah M, Thompson EW: Matrix metalloproteinase 13-deficient mice are resistant to osteoarthritic cartilage erosion but not chondrocyte hypertrophy or osteophyte development. Arthritis Rheum 2009, 60:3723-3733

10. Mellor J: Dynamic nucleosomes and gene transcription. Trends Genet 2006, 22:320-329

11. Felsenfeld G: Chromatin unfolds. Cell 1996, 86:13-19

12. Paranjape SM, Kamakaka RT, Kadonaga JT: Role of chromatin structure in the regulation of transcription by RNA polymerase II. Annu Rev Biochem 1994, 63:265-297

13. Marks PA, Miller T, Richon VM: Histone deacetylases. Curr Opin Pharmacol 2003, 3:344-351

14. Blanchard F, Chipoy C: Histone deacetylase inhibitors: new drugs for the treatment of inflammatory diseases? Drug Discov Today 2005, 10: 197-204

15. Adcock IM: HDAC inhibitors as anti-inflammatory agents. $\mathrm{Br} \mathrm{J}$ Pharmacol 2007, 150:829-831

16. Alcaraz MJ, Megias J, Garcia-Arnandis I, Clerigues V, Guillen MI: New molecular targets for the treatment of osteoarthritis. Biochem Pharmacol 2010, 80:13-21

17. Chen WP, Bao JP, Hu PF, Feng J, Wu LD: Alleviation of osteoarthritis by Trichostatin A, a histone deacetylase inhibitor, in experimental osteoarthritis. Mol Biol Rep 2010, 37:3967-3972

18. Akhtar N, Makki MS, Haqqi TM: MicroRNA-602 and microRNA-608 regulate sonic hedgehog expression via target sites in the coding region in human chondrocytes. Arthritis Rheumatol 2015, 67:423-434

19. Haseeb A, Makki MS, Haqqi TM: Modulation of ten-eleven translocation 1 (TET1), isocitrate dehydrogenase (IDH) expression, alphaketoglutarate (alpha-KG), and DNA hydroxymethylation levels by interleukin-1beta in primary human chondrocytes. J Biol Chem 2014, 289:6877-6885

20. Heinrich PC, Behrmann I, Haan S, Hermanns HM, Muller-Newen G, Schaper F: Principles of interleukin (IL)-6-type cytokine signalling and its regulation. Biochem J 2003, 374:1-20

21. Livak KJ, Schmittgen TD: Analysis of relative gene expression data using real-time quantitative PCR and the 2(-delta delta $\mathrm{C}(\mathrm{T})$ ) method. Methods 2001, 25:402-408 
22. Makki MS, Heinzel T, Englert C: TSA downregulates Wilms tumor gene 1 (Wt1) expression at multiple levels. Nucleic Acids Res 2008, 36:4067-4078

23. Legendre F, Bogdanowicz P, Boumediene K, Pujol JP: Role of interleukin 6 (IL-6)/IL-6R-induced signal transducers and activators of transcription and mitogen-activated protein kinase/extracellular. J Rheumatol 2005, 32:1307-1316

24. Makki MS, Haseeb A, Haqqi TM: MicroRNA-9 promotion of interleukin-6 expression by inhibiting monocyte chemoattractant protein-induced protein 1 expression in interleukin-1 $\beta$-stimulated human chondrocytes. Arthritis Rheumatol 2015, 67:2117-2128

25. Grabiec AM, Krausz S, de Jager W, Burakowski T, Groot D, Sanders ME, Prakken BJ, Maslinski W, Eldering E, Tak PP, Reedquist KA: Histone deacetylase inhibitors suppress inflammatory activation of rheumatoid arthritis patient synovial macrophages and tissue. J Immunol 2010, 184:2718-2728

26. Falkenberg KJ, Johnstone RW: Histone deacetylases and their inhibitors in cancer, neurological diseases and immune disorders. Nat Rev Drug Discov 2014, 13:673-691

27. Hong S, Derfoul A, Pereira-Mouries L, Hall DJ: A novel domain in histone deacetylase 1 and 2 mediates repression of cartilage-specific genes in human chondrocytes. FASEB J 2009, 23:3539-3552

28. Lu J, Sun Y, Ge Q, Teng H, Jiang Q: Histone deacetylase 4 alters cartilage homeostasis in human osteoarthritis. BMC Musculoskelet Disord 2014, 15:438

29. Higashiyama R, Miyaki S, Yamashita S, Yoshitaka T, Lindman G, Ito Y, Sasho T, Takahashi K, Lotz M, Asahara H: Correlation between MMP-13 and HDAC7 expression in human knee osteoarthritis. Mod Rheumatol 2010, 20:11-17
30. Culley KL, Hui W, Barter MJ, Davidson RK, Swingler TE, Destrument AP, Scott JL, Donell ST, Fenwick S, Rowan AD, Young DA, Clark IM: Class I histone deacetylase inhibition modulates metalloproteinase expression and blocks cytokine-induced cartilage degradation. Arthritis Rheum 2013, 65:1822-1830

31. Joosten LA, Leoni F, Meghji S, Mascagni P: Inhibition of HDAC activity by ITF2357 ameliorates joint inflammation and prevents cartilage and bone destruction in experimental arthritis. Mol Med 2011, $17: 391-396$

32. Nasu Y, Nishida K, Miyazawa S, Komiyama T, Kadota Y, Abe N, Yoshida A, Hirohata S, Ohtsuka A, Ozaki T: Trichostatin A, a histone deacetylase inhibitor, suppresses synovial inflammation and subsequent cartilage destruction in a collagen antibody-induced arthritis mouse model. Osteoarthritis Cartilage 2008, 16:723-732

33. Lim H, Kim HP: Matrix metalloproteinase- 13 expression in IL-1betatreated chondrocytes by activation of the $\mathrm{p} 38 \mathrm{MAPK} / \mathrm{c}-\mathrm{Fos} / \mathrm{AP}-1$ and JAK/STAT pathways. Arch Pharm Res 2011, 34:109-117

34. Mengshol JA, Vincenti MP, Coon CI, Barchowsky A, Brinckerhoff CE: Interleukin-1 induction of collagenase 3 (matrix metalloproteinase 13) gene expression in chondrocytes requires p38, c-Jun N-terminal kinase, and nuclear factor kappaB: differential regulation of collagenase 1 and collagenase 3. Arthritis Rheum 2000, 43:801-811

35. Zhong HM, Ding QH, Chen WP, Luo RB: Vorinostat, a HDAC inhibitor, showed anti-osteoarthritic activities through inhibition of iNOS and MMP expression, p38 and ERK phosphorylation and blocking NFkappaB nuclear translocation. Int Immunopharmacol 2013, 17:329-335

36. Heinrich PC, Behrmann I, Muller-Newen G, Schaper F, Graeve L: Interleukin-6-type cytokine signalling through the gp130/Jak/STAT pathway. Biochem J 1998, 334:297-314 